\title{
Enhanced EHD and Electrostatic Propulsion Devices Based on Polarization Effect Using Asymmetrical Metal Structure
}

\author{
Taku Saiki \\ Department of Electrical and Electronic Engineering, Faculty of Engineering Science, Kansai University, Suita, Japan
}

Email address:

tsaiki@kansai-u.ac.jp

To cite this article:

Taku Saiki. Enhanced EHD and Electrostatic Propulsion Devices Based on Polarization Effect Using Asymmetrical Metal Structure. Journal of Electrical and Electronic Engineering. Vol. 3, No. 4, 2015, pp. 76-86. doi: 10.11648/j.jeee.20150304.13

\begin{abstract}
Electro hydro dynamic (EHD) and electrostatic propulsion devices were developed in the 1920s by Thomas Townsend Brown. One such device, called a "lifter", has no moving parts and, in the air, operates on electrical energy. It is a fashionable device and has a very simple structure, basically consisting of a narrow wire electrode and a large, flat one. However, it has a low ratio of propulsion force to unit electrical input power. According to theory, the propulsion force it generates depends on the interaction between the ion density of the ionized air and the charges on the surface of the large electrode. EHD and electrostatic propulsion models using the polarization effect are proposed to improve the ratio of the propulsion force to unit electrical input power. The propulsion device generates propulsion force through the use of an asymmetrical metal structure with charges generated by the polarization effect. The propulsion force the new devices generated for the same electric energy was 5.7 times higher than that of a basic type lifter owing to additional propulsion force being generated by the maximum polarization effect in the experiments. It was found that combining other effects with this polarization effect results in the ratio of generated propulsion force to electric power being close to $100 \mathrm{~N} / \mathrm{kW}$ when the electric power is high. This value is as high as that of a helicopter. We also performed numerical analysis was also performed for capacitances and charges for various kinds of EHD and electrostatic propulsion devices. An optimized system was developed and is discussed in this paper.
\end{abstract}

Keywords: EHD, Electrostatic Propulsion, Polarization, Electric Field, Charge, Electron

\section{Introduction}

A lifter that is made light in weight by using a high voltage supply floats in the air. For such lifters, only electrical energy is needed to obtain the propulsion force in the air. Normal propulsion devices such as rockets need materials to be propelled. Lifter-type propulsion devices have no moving parts as helicopters do, and can be expected to be used as Unmanned Aerial Vehicles (URVs) in the future [1].

The Biefeld-Brown effect was discovered by Paul Alfred Biefeld and Thomas Townsend Brown in the 1920s [2]. Brown also proposed and was awarded patents for a number of electro hydro dynamic (EHD) propulsion devices, such as lifters $[1,3]$. The principle, which is called ion craft, should be the same as that of the lifter. Many movies of lifters floating in the air can be seen on the Internet, and a number of papers on the theory of lifters to generate propulsion forces have been published[4-12]. After the Biefeld-Brown effect was published, many discussions on it were held. Mainly, the principles behind the effect were discussed and many theories to explain the generated propulsion forces were proposed. One view, that the effect can be explained by "generation of momentum by ion wind", was shown by the USA's National Renewable Energy Laboratory. However, recent rigid research indicates that the principle of the propulsion can be explained as the electrical forces between charges in ionic wind and the electrons on the large flat electrode[6-8]. Thus, it has been shown that the lifter propulsion is based on a principle that differs from conventional principals. Rockets work on the basis of the equation of motion.

The objectives in developing the propulsion devices are achieving 1) low propulsion force to electrical input energy, 2) low propulsion force per unit volume, and 3) low propulsion force per unit weight. Some papers $[12,13]$ have previously reported experimental results for improving the ratio of generated propulsion force to electric energy. Thus, at present, heavy, high voltage sources cannot be mounted on propulsion devices.

In this paper, I propose an improved model for the structure of the rounded electrode used to improve the charge density of ion wind. It is a model with a simplified small negative 
electrode to make the ion source small. In addition, propulsion devices using the polarization effect, such as a cascading model, were fabricated to improve the ratio of propulsion force to electrical input energy.

\section{Principle of EHD and Electrostatic Propulsion Devices}

Simply put, a lifter consists of a tin wire electrode and a large, flat electrode [1]. The principle for producing the propulsion force of a lifter and EHD propulsion devices was proposed in recent research activities shown in the literature $[8,11]$. An equation to calculate the generated propulsion force of a lifter is shown next in references [8] and [11]

$$
F=\int_{v} \rho E \cdot d v
$$

In the equation, $\mathrm{E}$ is the electric field generated from the charges on the large flat electrode and $v$ is the effective volume area in which ionized air flows near the large electrode. The equation for calculating $\rho$, the ion density near the large electrode in the air, is

$$
\rho=\frac{\mathrm{Id}}{\mu \mathrm{VA}} .
$$

Here, $\mathrm{I}$ is the current, $\mathrm{d}$ is the gap between the tin wire electrode and the large flat electrode, $\mu$ is the ion mobility in the atmosphere, $\mathrm{V}$ is the voltage between the electrodes, and $\mathrm{A}$ is the profile area of ionized air floating near the large electrode. It shows that all the generated ions do not interact with the large electrode. Here, if we set $\mathrm{E}=\mathrm{V} / \mathrm{d}$, eq. (1) is simplified and we obtain

$$
\mathrm{F}=\frac{\mathrm{Id}}{\mu}
$$

Here, I describe the meaning of eq. (1). The charges on the large electrode attract the ion wind by the electric field between the generated ions and the large flat electrode. As a result, propulsion force is produced. The observed current at the power supply results from the fact that ions exchange the electrons on the large electrode (interaction between the ion wind and the large flat electrode). Detailed numerical estimations of EHD fluids, space charge, and potential in the ionized air flow are shown in reference [5-9].

The ratio of generated power to electrical input power is given by

$$
\theta=\frac{F}{P}=\frac{I}{\mu E} .
$$

Below, I simply describe the calculation method for the capacitance between the electrodes and the charge on them. The method is shown in eq. (1), according to the principle for producing propulsion force. Evaluating the quantity of the charge on the electrode when a high voltage is applied results in improved propulsion force. However, the electrical charge is not always proportional to the propulsion forces the EHD devices generate.

In this work, I used a rounded electrode for the structure of the EHD device in order to 1) use the model as a unit module, 2) achieve uniform divergence of the ionized air in the radial direction, and 3) efficiently protect the ion density in the rounded electrode from degradation.

I used metal structure for the device to enhance 1) the charges on the electrodes owing to the polarization effect, 2) the emission of ions from the negative needle electrode, and 3) the propulsion force produced by the electrical field between the ion wind and the large electrode or between the ion wind and the polarization electrodes.

For the idea for polarization effect in EHD devices, one [1] of Brown's patents proposed that an EHD device in which a dielectric material is sandwiched between the electrodes produces high propulsion force. The details for this experiment differed from those given in Brown's patent in that propulsion forces are generated by metal electrodes for which an asymmetrical structure is used to produce the polarization effect.

Here, the needle electrode was connected to the negative and the large electrode to the positive voltage. The structure for generating ions was simplified.

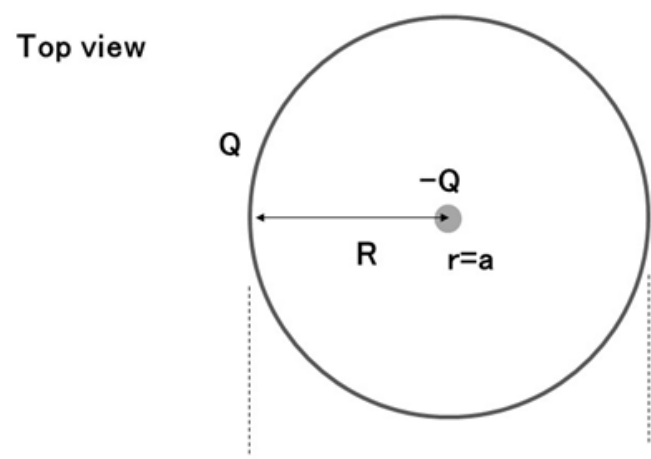

Side view

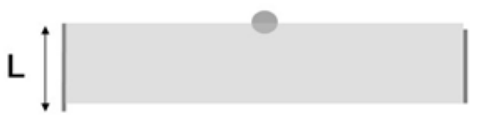

(a)

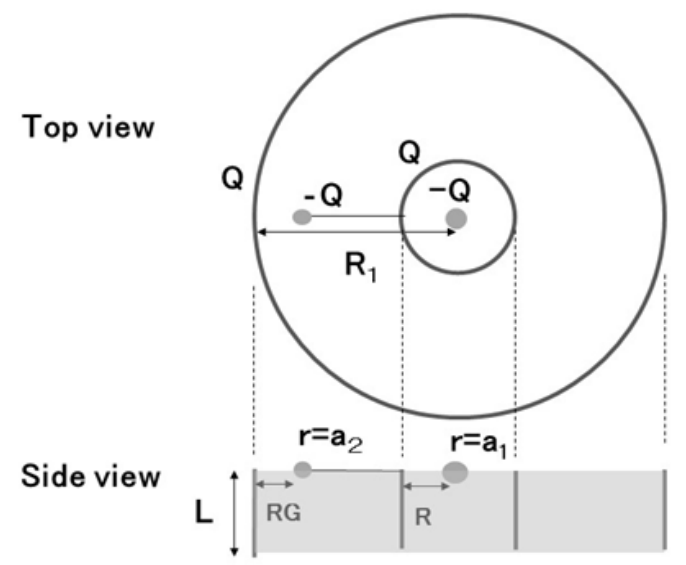

(b) 


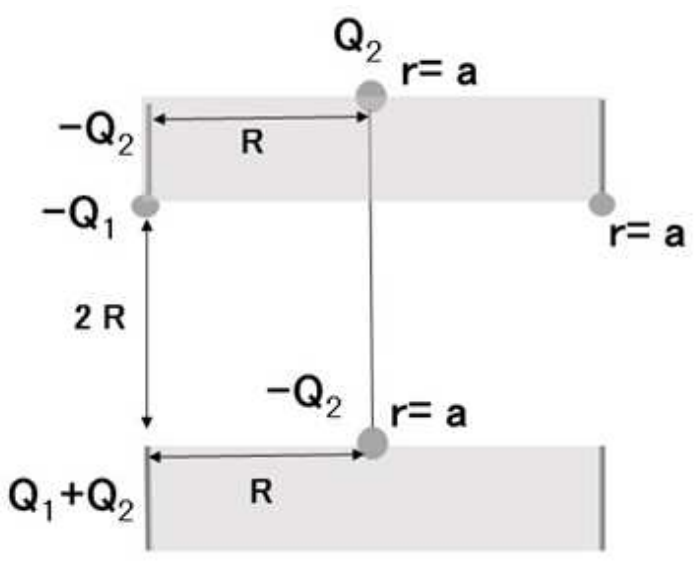

(c)

Fig. 1. Calculation models. (a) normal model 1a, (b) double ring model 1b, (c) polarization pole model $1 \mathrm{c}$

For the model shown in Fig. 1(a) I used a rounded electrode, in contrast to the conventional lifter that uses flat plate electrodes. Here, I neglected ion flow because its calculation is very complicated. The equation to calculate the electrical potential on the radial axis $r$ for model $1 \mathrm{a}$ is

$$
\mathrm{V}(\mathrm{r})=\frac{-\mathrm{Q}}{4 \pi \varepsilon_{0}} \cdot\left[\frac{1}{\mathrm{r}}-\frac{\ln \left[\frac{\mathrm{L}+\left(\mathrm{L}^{2}+(\mathrm{R}-\mathrm{r})^{2}\right)^{1 / 2}}{\mathrm{R}-\mathrm{r}}\right]}{\mathrm{L}}\right] .
$$

Here, $\mathrm{L}$ is the width of the large electrode and $\mathrm{R}$ is the radius of the large electrode. Thus, the equation for the electrical potential difference $V_{a b}$ between both electrodes is

$$
\mathrm{V}_{\mathrm{ab}}=\frac{\mathrm{Q}}{4 \pi \varepsilon_{0}} \cdot\left[\frac{1}{\mathrm{a}}-\frac{1}{\mathrm{~b}}+\ln \left[\frac{\mathrm{R}-\mathrm{a}}{\mathrm{R}-\mathrm{b}} \cdot \frac{\mathrm{L}+\left(\mathrm{L}^{2}+(\mathrm{R}-\mathrm{b})^{2}\right)^{1 / 2}}{\mathrm{~L}+\left(\mathrm{L}^{2}+(\mathrm{R}-\mathrm{a})^{2}\right)^{1 / 2}}\right]\right]
$$

Here, $0<\mathrm{a}, \mathrm{b}<\mathrm{R}$, and it is assumed that $\mathrm{b}$ is very close to $\mathrm{R}$. The equation for the capacitance between the electrodes for model 1a is

$$
\mathrm{C}_{\mathrm{ab}}=\frac{4 \pi \varepsilon_{0}}{\left[\frac{1}{\mathrm{a}}-\frac{1}{\mathrm{~b}}+\ln \left[\frac{\mathrm{R}-\mathrm{a}}{\mathrm{R}-\mathrm{b}} \cdot \frac{\mathrm{L}+\left(\mathrm{L}^{2}+(\mathrm{R}-\mathrm{b})^{2}\right)^{1 / 2}}{\mathrm{~L}+\left(\mathrm{L}^{2}+(\mathrm{R}-\mathrm{a})^{2}\right)^{1 / 2}}\right]\right]}
$$

The third term in eq. (6) can be neglected because it is very small compared to the first term. If $L>>R$, eq. (7) is more simplified and changed to

$$
\mathrm{C}_{\mathrm{ab}}=4 \pi \varepsilon_{0} \frac{\mathrm{b} \cdot \mathrm{a}}{\mathrm{b}-\mathrm{a}} .
$$

For calculating model $1 \mathrm{~b}$, there is one metal rod electrode to produce polarization connected directly to inner electrode. RG is the gap length as shown in Fig. 1(b). RG is very much shorter than L. Here, $\mathrm{C}_{\mathrm{a} 2 \mathrm{~b} 2}$ is generated capacitance governed by eq. (7) to set the space gap and $\mathrm{C}_{\mathrm{a} 1 \mathrm{~b} 1}$ is the capacitance between the negative needle electrode and the inner radial electrode governed by eq. (8). The total capacitance for model $1 \mathrm{~b}$ is determined by calculating the series connection of $\mathrm{C}_{\mathrm{a} 1 \mathrm{~b} 1}$ and $\mathrm{C}_{\mathrm{a} 2 \mathrm{~b} 2}$. The equation for calculating the capacitance for model $1 \mathrm{~b}$ is

$$
\mathrm{C}_{2}=\frac{\mathrm{C}_{\mathrm{a} 1 \mathrm{~b} 1} \cdot \mathrm{C}_{\mathrm{a} 2 \mathrm{~b} 2}}{\mathrm{C}_{\mathrm{a} 1 \mathrm{~b} 1}+\mathrm{C}_{\mathrm{a} 2 \mathrm{~b} 2}} .
$$

If the number of metal rods needed to generate polarization is $\mathrm{n}$, the capacitance is rewritten as

$$
\mathrm{C}_{2}{ }^{\prime}=\mathrm{nC}_{2} \text {. }
$$

In the case of model $1 \mathrm{c}$, the capacitance between the upper and lower ring electrodes is

$$
\mathrm{C}_{0}=2 \pi \varepsilon_{0} \frac{2 \pi \mathrm{R}}{\ln (2 \mathrm{R} / \mathrm{a})}
$$

The capacitance generated by using a center electrode to generate polarization is the same as that for model $1 \mathrm{~b}$. The capacitance $\mathrm{C}_{2}$ is derived by calculating the series connection of two $C_{a b}$ shown in eq. (7). The total capacitance $C_{3}$ for model $1 \mathrm{c}$, derived by calculating the parallel connection of $\mathrm{C}_{2}$ and $\mathrm{C}_{0}$, is

$$
\mathrm{C}_{3}=\mathrm{C}_{0}+\mathrm{C}_{2} \text {. }
$$

It is clear that eq. (12) and eq. (9) indicate increased capacitances in each of the propulsion models.

Here I return to the discussion about the propulsion force. For model $1 \mathrm{~b}$, the generated propulsion force obtained by reconsidering eq. (1) is

$$
F_{2}=\int_{v}\left(\rho_{1} E_{1}+\rho_{2} E_{2}\right) \cdot d v
$$

Here, $E_{1}$ is the electric field producing the charges on the inner radial ring to generate polarization, $E_{2}$ is the electric field producing the charges on the large outer radial electrode, $\rho_{1}$ is the ion density near the inner radial ring to generate polarization, and $\rho_{2}$ is the ion density near the large outer radial electrode.

For model 1c, the generated propulsion force obtained by reconsidering eq. (1) is

$$
F_{3}=\int_{v}\left[\rho_{3} E_{3}+\rho_{4}\left(E_{2}+E_{4}\right)\right] \cdot d v .
$$

Here, $E_{3}$ is the electric field producing the charge $-\mathrm{Q}_{2}$ on the upper ring, $\mathrm{E}_{4}$ is the electric field producing the charges $\mathrm{Q}_{2}$ on the lower ring electrode, $\rho_{3}$ is the ion density near the upper radial electrode (the ions are produced at the edge of the polarization rod at the center of the module), and $\rho_{4}$ is the ion 
density near the lower ring electrode (the ions are produced at the edge of the upper ring electrode). Here, the polarity of $\rho_{3}$ is positive and that of $\rho_{4}$ is negative. From eq. (13) and eq. (14), comparing them to eq. (1), it is concluded that the propulsion forces that both model $1 \mathrm{~b}$ and model $1 \mathrm{c}$ generate should be modified. Here, it should be noted that it is not always necessary to exchange the electrons between the electrodes when the forces are produced. In other words, the current is not needed to generate the propulsion forces from the polarization electrodes.

Finally, the ratios of generated power to electrical input power for model $1 \mathrm{~b}$ and $1 \mathrm{c}$ are given by $\theta_{2}=\mathrm{F}_{2} / \mathrm{P}$, and $\theta_{3}=\mathrm{F}_{3} / \mathrm{P}$, respectively.

\section{Experimental Setup}

\subsection{Propulsion Model}

Three types of model A units, which were actually fabricated in this work, are shown in Fig. 2. These models, which produce propulsion force, can be set in series or parallel I also fabricated two other types of models. In one, the ion wind flows downward in the ring electrode. In the other, the ion wind expands in the radial direction and flows outside the ring electrode. In all the A model units, the ion wind flows inside the ring electrode (Fig. 2). The distance between the negative needle electrode and the positive large ring electrode in the vertical direction was set to be $4 \mathrm{~cm}$ in each case.

\section{Negative electrode}

\section{Side view}

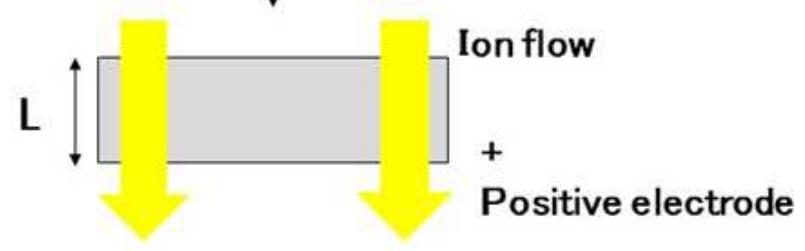

Top view

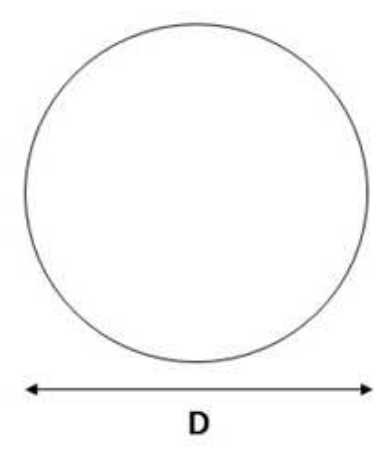

(a)

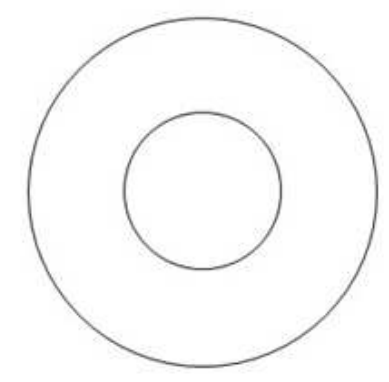

Round

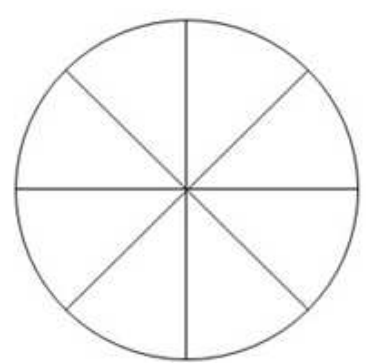

Radial

(c)

Fig. 2. Enhanced EHD and electrostatic propulsion devices. Model A units: (a) normal Aa, (b) with added inner ring electrode Ab, (c) with added radial electrode Ac.

Table 1. Model A parameters.

\begin{tabular}{lllll}
\hline Model & $\begin{array}{l}\text { Diameter } \\
\text { D }(\mathbf{c m})\end{array}$ & $\begin{array}{l}\text { Height } \\
\mathbf{L}(\mathbf{c m})\end{array}$ & $\begin{array}{l}\text { Diameter of } \\
\text { Inner Ring } \\
\mathbf{D}_{\mathbf{1}}(\mathbf{c m})\end{array}$ & $\begin{array}{l}\text { Height of Inner } \\
\text { Ring } \mathbf{L}_{\mathbf{1}}(\mathbf{c m})\end{array}$ \\
\hline $\mathrm{Aa} 1$ & 8 & 3 & - & - \\
$\mathrm{Aa} 2$ & 16 & 5 & - & - \\
$\mathrm{Ab}$ & 16 & 5 & 8 & 5 \\
\hline
\end{tabular}

The aluminum foils used as large electrodes were $10 \mu \mathrm{m}$ in thickness. To improve the capacitance of the devices, various kinds of electrodes were added inside the large ring electrodes. The Aa model has a single ring electrode, the $\mathrm{Ab}$ model has another ring electrode inside the outer ring electrode, and the Ac model has a star electrode inside the outer ring electrode. These models were used to conduct an experiment to produce high propulsion forces. The size parameters are shown in Table 1. The weights of the model Aa1, Aa2 and Ab were $0.6 \mathrm{~g}$,
$1.5 \mathrm{~g}$, and $1.8 \mathrm{~g}$, respectively.

The devices were set in series to enhance the propulsion force for the same electrical input power. In Fig. 3 depicting model B1, the cascading of two devices is shown at the left side and the cascading of $\mathrm{n}$ devices is shown at the right. Model at the left side in Fig. 3 was used in this experiment. For the model at the left side, the distance between the upper ring electrode and the lower ring electrode was set to be $3 \mathrm{~cm}$. A wire was connected on the upper ring electrode and the end was closed to the lower ring electrode with a gap of $1 \mathrm{~cm}$. It was normally difficult to connect the devices in series because in some cases the propulsion forces disappeared due to ion flow or the positive and negative electrode settings. The upper ring electrode generates polarization. Negative charges are collected at the edge of the wire connected on the upper ring electrode. The weights of the model B1 was1.1g. 
OCascading Two module

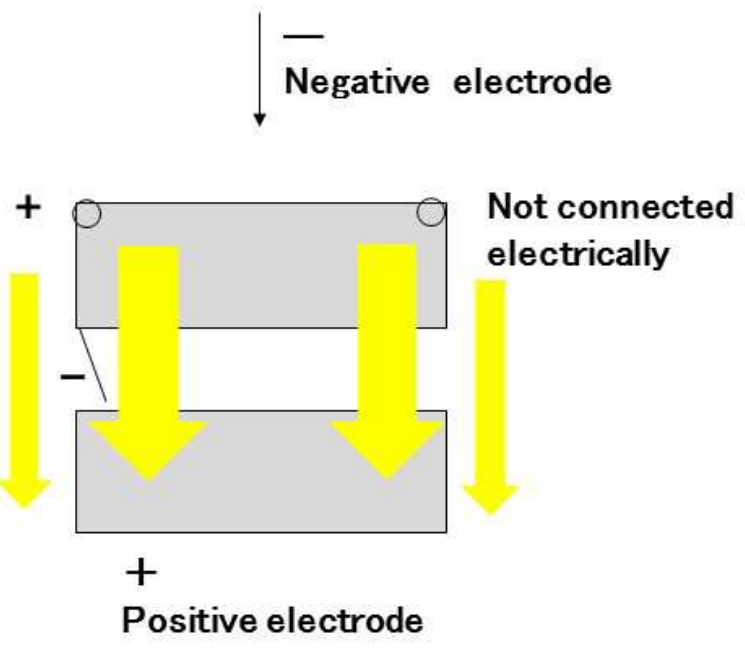

\section{OCascading \\ $\mathrm{N}$ module}

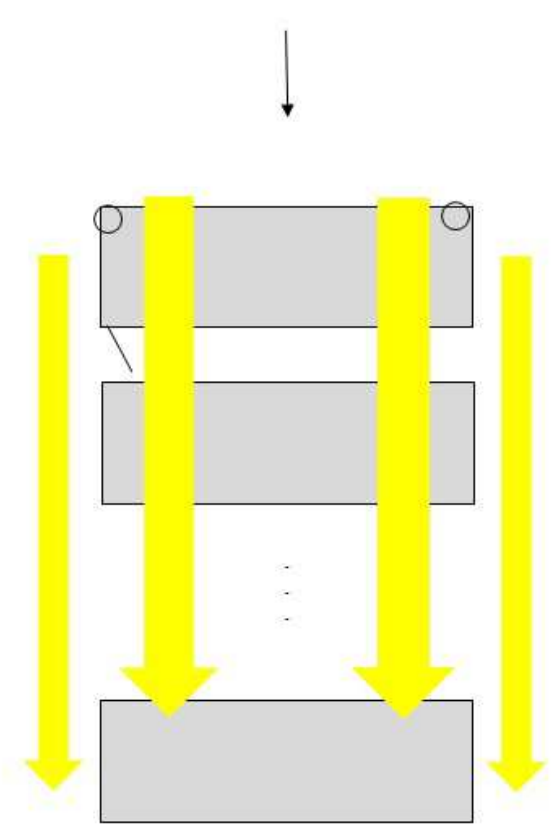

Fig. 3. Enhanced EHD and electrostatic propulsion devices. Model B1.

Model B2 (Fig. 4) has a metal wire at the center of the module to generate polarization. Positive ions are produced from the upper edge of the wire, generating a propulsion force to the upper ring electrode by the positive ion flow. Thus, two propulsion forces, which work on both the upper ring electrode and the lower ring electrode, are generated. The weights of the model B2 was $3.2 \mathrm{~g}$. The model is adapted to Fig. 1(c).

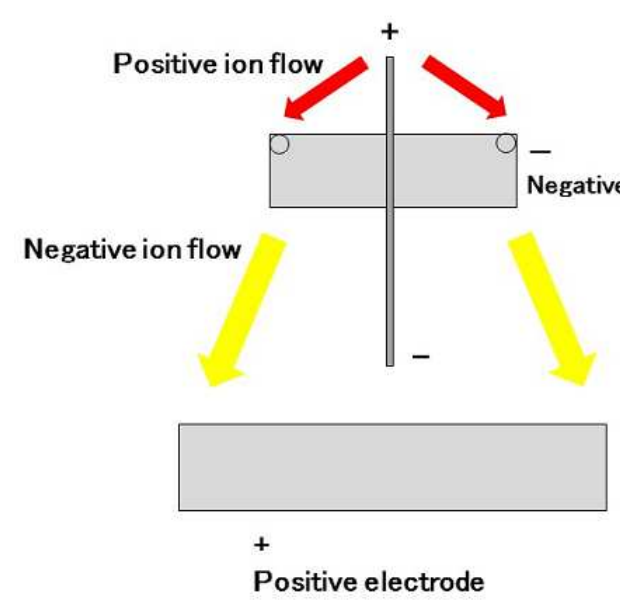

(a)

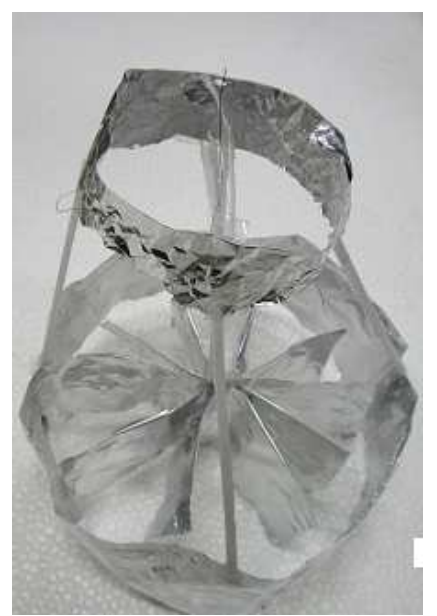

(b)

Fig. 4. Enhanced EHD and Electrostatic propulsion devices Model B2. (a) Structure, (b) photo image.

In model B3 (Fig. 5), the ion wind flows in the radial direction. Enhanced propulsion force is produced by the polarization effect. The model is adapted to Fig. 1(b). Four small plate electrodes are set in the radial direction inside the outer large ring electrode. Negative ions generated from the upper negative needle electrode are trapped the outer large ring electrode and finally the current is supplied from a high voltage source. The structure is proposed because an EHD generator should be set in the center of the device. In the future such generators will use a weakly ionized plasma (such as a flame) to generate an ultra-high voltage [15].The weights of the model B3 was $3.8 \mathrm{~g}$. 
Side View

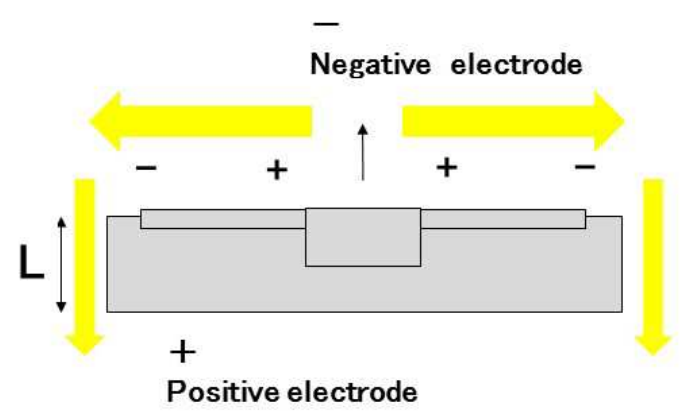

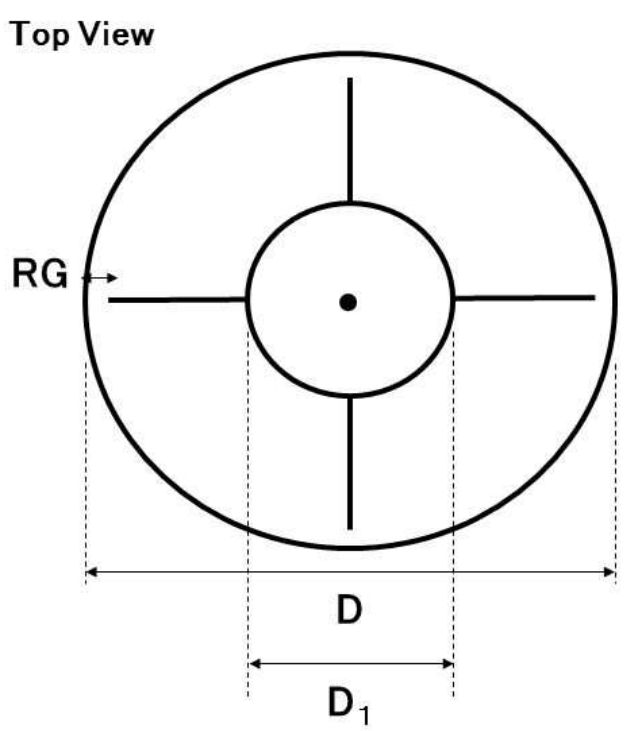

(a)

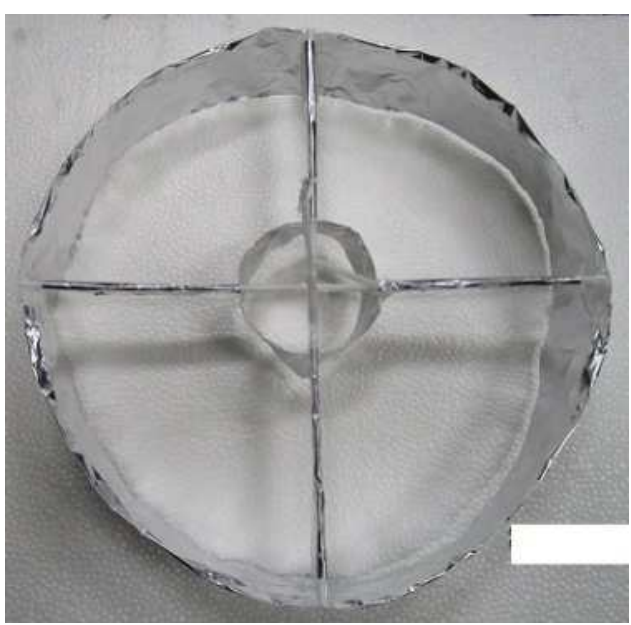

(b)

Fig. 5. Enhanced EHD and Electrostatic propulsion devices Model B3. (a) Structure, (b) photo image.

The structure parameters of model B are shown in Table 2.

Table 2. Model B parameters.

\begin{tabular}{|c|c|c|c|c|c|c|}
\hline Model & $\begin{array}{l}\text { Diameter of Ring } \\
\text { D(cm) }\end{array}$ & $\begin{array}{l}\text { Height L } \\
\text { (cm) }\end{array}$ & $\begin{array}{l}\text { Diameter of Inner } \\
\text { (Upper) Ring } D_{1}(\mathrm{~cm})\end{array}$ & $\begin{array}{l}\text { Height of Inner (Upper) } \\
\text { Ring } L_{1}(\mathrm{~cm})\end{array}$ & Gap (cm) & $\begin{array}{l}\text { Distance between } \\
\text { Top and Bottom Rings }(\mathrm{cm})\end{array}$ \\
\hline B1 & 8 & 3 & 8 & 3 & 1 & 3 \\
\hline B2 & 16 & 5 & 8 & 3 & $\begin{array}{l}\text { Upper } 2 \\
\text { Lower } 3\end{array}$ & 9 \\
\hline B3 & 32 & 5 & 8 & 3 & 1 & - \\
\hline
\end{tabular}

\subsection{Measurement of Propulsion Force}

The instrument used to measure the propulsion forces of the EHD devices is shown in Fig. 6.

The propulsion devices were connected to a DC high voltage generator. A six stage Cockcroft Walton circuit was used to generate the voltage. A sinewave generator was connected to the Cockcroft Walton circuit. In this experiment, a DC power supply (PR36-3A, TEXIO, Japan) was used to generate a sinewave with a high output voltage and $25 \mathrm{KHz}$ frequency. The output voltage was evaluated by using a voltage tester (CD731a, SANWA, Japan) and a high voltage probe (HV-60SANWA, SANWA, Japan). The maximum measured output voltage of the Cockcroft Walton circuit was $48 \mathrm{kV}$. The current was evaluated by connecting $22 \mathrm{k}$ ohm resistors in series to the propulsion devices and measuring the voltage. A dielectric plastic rod with a diameter of $1.0 \mathrm{~cm}$ was set on a gravimeter (ACS-20, ASONE, Japan). The propulsion force was measured by reading the decrease in the weight. 


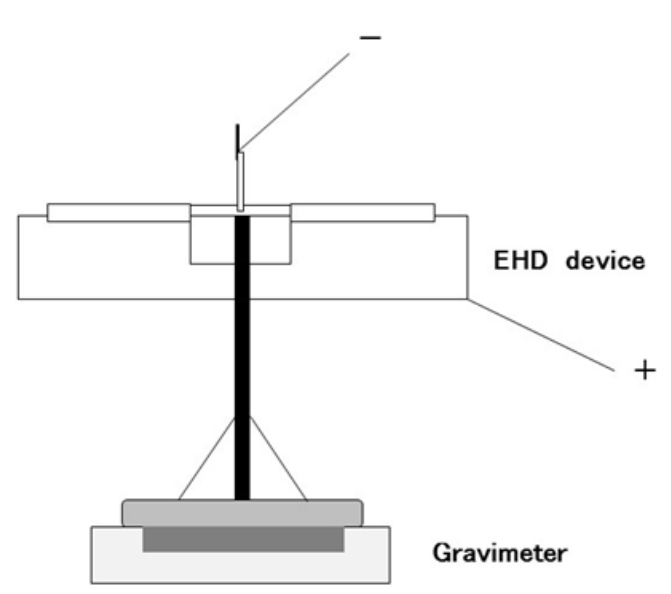

Fig. 6. Instrument for measuring propulsion force of the enhanced EHD propulsion devices.

\section{Results}

\subsection{Calculated Capacitance and Charge on Electrodes}

The calculated results on the capacitance of model 1a are shown as a function of $\mathrm{L}$ in Fig. 7. Here, $\mathrm{R}$ is set to be $4 \mathrm{~cm}$ and is fixed at that length. It was assumed that the calculating space is in the vacuum.

The results clarified that the capacitance for model 1a, shown in Fig. 1(a), the capacitance increased gradually and saturated as L increased.

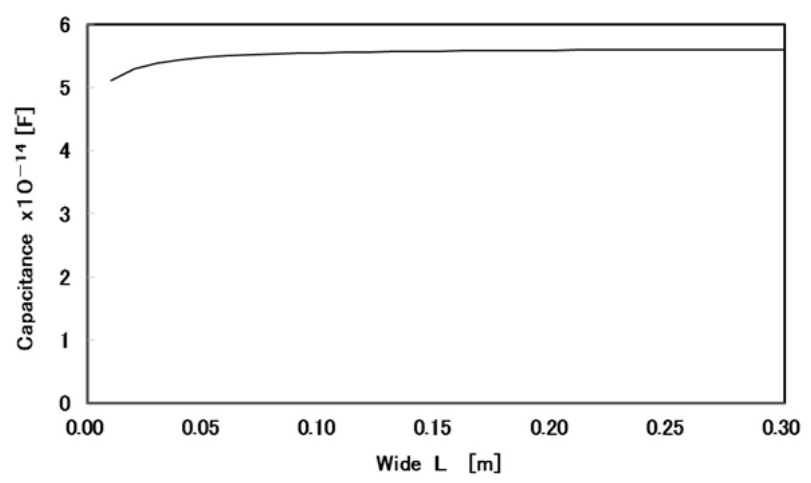

Fig. 7. Calculated capacitance for model 1a as a function of L.

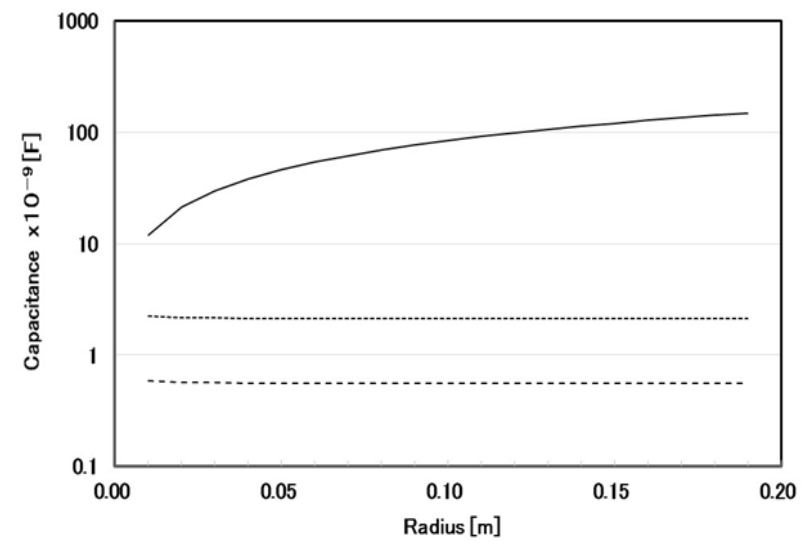

Fig. 8. Calculated capacitance results for each propulsion model.
The calculated results of the capacitance for each model are shown in Fig. 8. The dashed line shows the results for model $1 \mathrm{a}$, the dotted line shows the results for model $1 \mathrm{~b}$, and the solid line shows the results for model 1c. a and a1 are all set to be $0.005[\mathrm{~m}]$. b was set to be R-0.005[m]. To simplify the calculation, L was set to $0.03[\mathrm{~m}]$, assuming that $\mathrm{R} 1$ is adequately longer than $R$. $n$ was set to be 4 and $R G$ was set to be $1 \mathrm{~cm}$ in the calculation. The capacitances for model $1 \mathrm{a}$ and $1 \mathrm{~b}$ degraded as $\mathrm{R}$ increased. However, the capacitances for model 1c increased as $\mathrm{R}$ increased due to the structure.

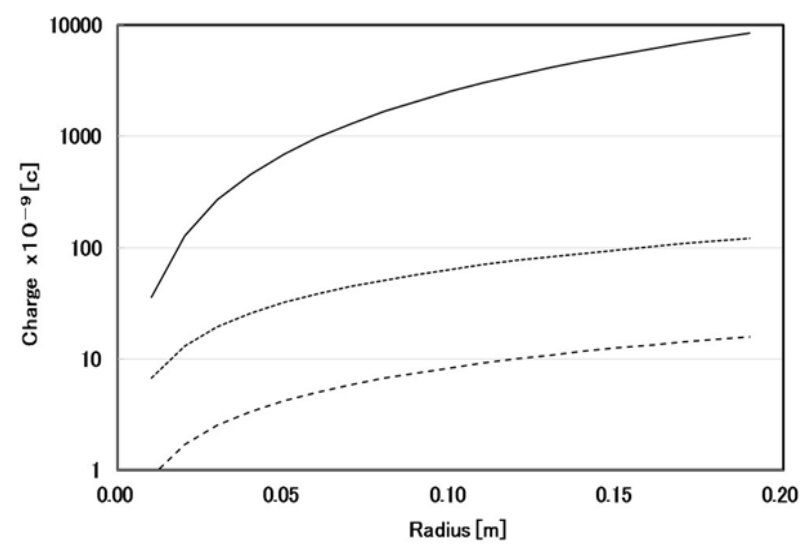

Fig. 9. Calculated charge results.

The calculated results for the total charges on the large electrode are shown in Fig. 9. The dashed line shows the results for model 1a. The dotted line shows the results for model $1 \mathrm{~b}$, and the solid line shows the results for model $1 \mathrm{c}$. For the input voltage to the propulsion devices, I consider the discharge property between the needle and flat electrodes, i.e., the limit of $15[\mathrm{kV}]$ per $\mathrm{cm}$ for the discharge determined [14]. Thus, the maximum input voltage was given by an equation of $15 \times \mathrm{R}[\mathrm{kV}]$. For model 1c, the calculated charge was obtained by adding the charge on the upper ring electrode to that on the lower ring electrode. For model $1 \mathrm{~b}$, it was obtained by adding the charge on the inner ring electrode to that on the outer large ring electrode. The charges for model $1 \mathrm{a}, 1 \mathrm{~b}$, and $1 \mathrm{c}$ increased as $\mathrm{R}$ increased. The charges for model $1 \mathrm{c}$ were the largest for all the models.

\subsection{Measured Propulsion Force}

The results obtained for the measured propulsion force for model Aa1, Aa2, and $\mathrm{Ab}$ are shown in Fig. 10. The black circle dots show the results for model Aa2. The white circle dots shows the results for model Aa1. The white square dots shows the results for model $\mathrm{Ab}$. The data shown by the dots in Fig. 10(a) is consistent with that shown by the dots in Fig. 10(b). The calculated electrical input power in Fig. 10(b) was obtained by multiplying the measured current data. When the input voltages were 22 and $26 \mathrm{kV}$, the measured current levels were 0.15 and $0.26 \mathrm{~mA}$, respectively.

When the electrical input power was $8 \mathrm{~W}$, it can be seen that the force that model Aa2 generated was 2.5 times higher than that of model Aa1. The maximum generated force was $1.8 \mathrm{~g}$ at an input voltage of $43 \mathrm{kV}$. However, the generated 
force was gradually saturated as the input voltage and the electrical input power increased. In another experiment, the force measured for model $\mathrm{Aa} 2$ for $\mathrm{L}=10 \mathrm{~cm}$ was $2.0 \mathrm{~g}$ at an input voltage of $43 \mathrm{kV}$. In spite of using a long $\mathrm{L}$, the generated force was only slightly improved.

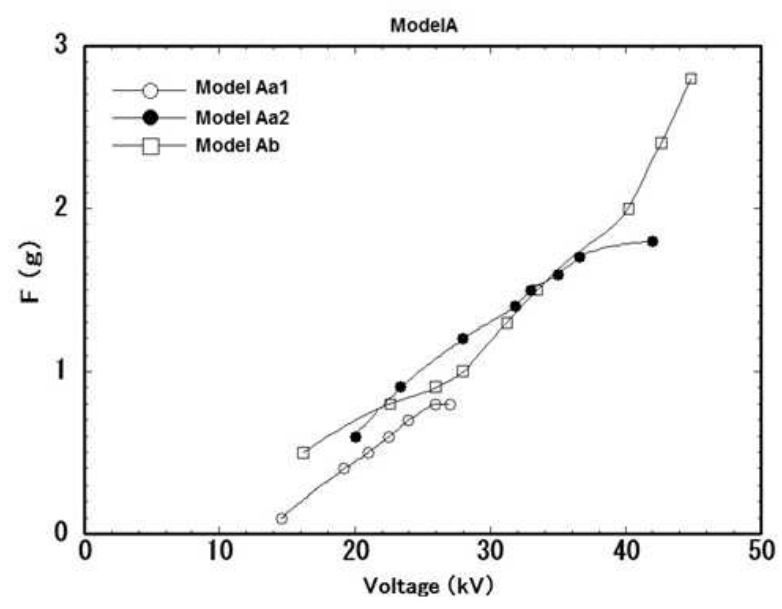

(a)

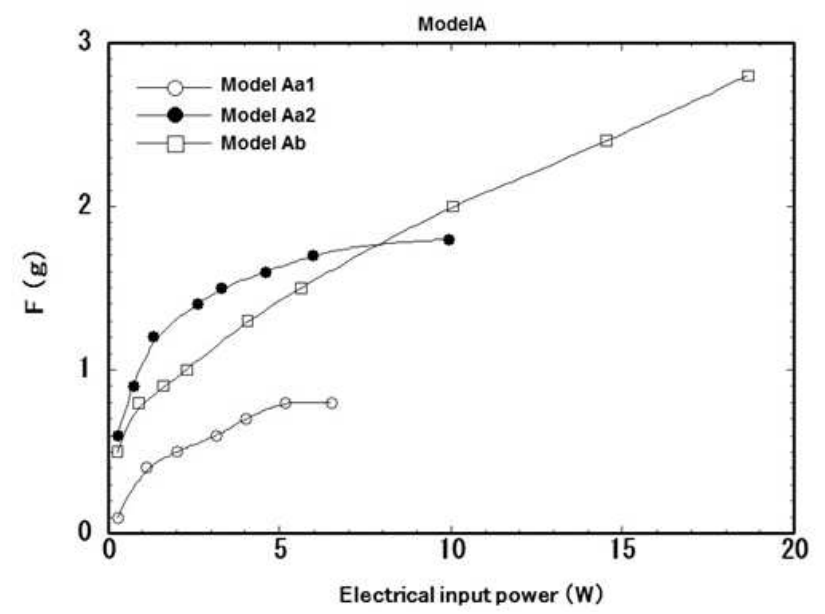

(b)

Fig. 10. Measured propulsion force results for model Aa1, Aa2, and Ab. (a) Voltage-force property, (b) Power-force property.

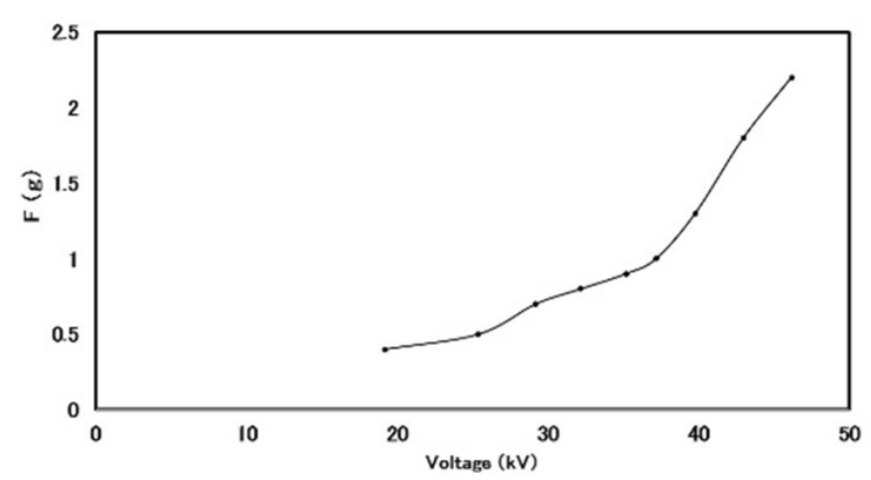

(a)

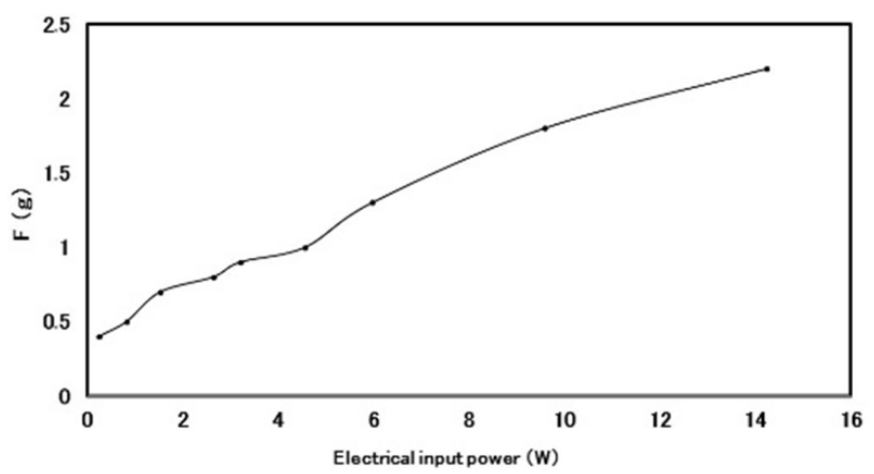

(b)

Fig. 11. Measured propulsion force results for model B1. (a) Voltage-force property, (b) Power-force property.

When the electrical input voltage was $44 \mathrm{kV}$, it can be seen that the propulsion force that model $\mathrm{Ab}$ generated was 1.6 times higher than that of model Aa1. The maximum generated force was $2.8 \mathrm{~g}$ at an input voltage of $44 \mathrm{kV}$. However, the generated force was not saturated as the input voltage and the electrical input power increased. In another experiment, in which a star electrode and two inner multi-rings were added to model Aa2, the maximum generated forces for them were 2.3 and $3.2 \mathrm{~g}$, respectively.

The measured propulsion force results obtained for model B1 are shown in Fig. 11. If the generated power values shown in Fig. 10(a) are compared with those shown in Fig. 11(a), when the electrical input voltage was $46 \mathrm{kV}$, it can be seen that the force that model B1 generated was 3 times higher than that of model Aa1. The maximum generated force was $2.3 \mathrm{~g}$ at an input voltage of $44 \mathrm{kV}$. The generated force markedly increased at an input voltage of $38 \mathrm{kV}$ as shown in Fig. 11(a).
The measured propulsion force results obtained for model B2 are shown in Fig. 12. If the generated power values shown in Fig. 10(a) are compared with those shown in Fig. 12(a), when the electrical input voltage was $43 \mathrm{kV}$, it can be seen that the force that model B2 generated was 2 times higher than that of model Ac. The maximum generated force was $3.6 \mathrm{~g}$ at an input voltage of $43 \mathrm{kV}$. The generated force increased as a function of a value close to the square of the input voltage as shown in Fig. 12(a).

Measured propulsion force results obtained for model B3 are shown in Fig. 13. The maximum generated force was $5.1 \mathrm{~g}$ at an input voltage of $48 \mathrm{kV}$. The generated force increased as a function of a value close to the square of the input voltage as shown in Fig. 13(a). If the generated power values shown in Fig. 10(a) are compared with those shown in Fig. 13(a), it can be seen that the maximum generated force that model B3 generated was 6.4 times higher than that of model Aa1. 


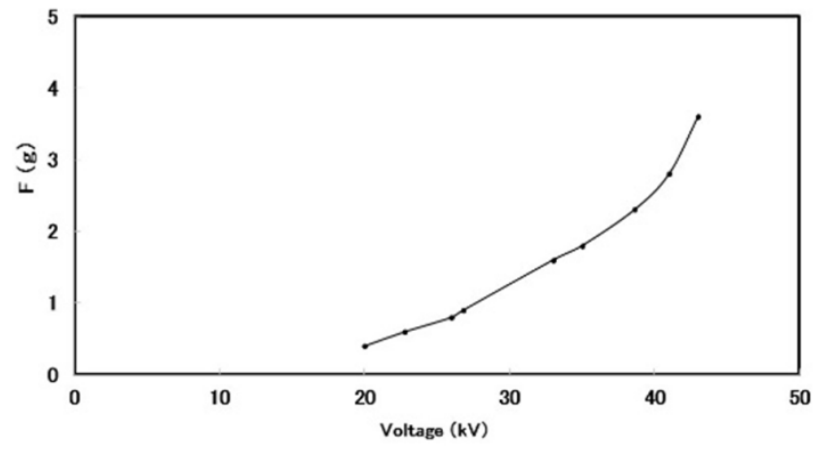

(a)

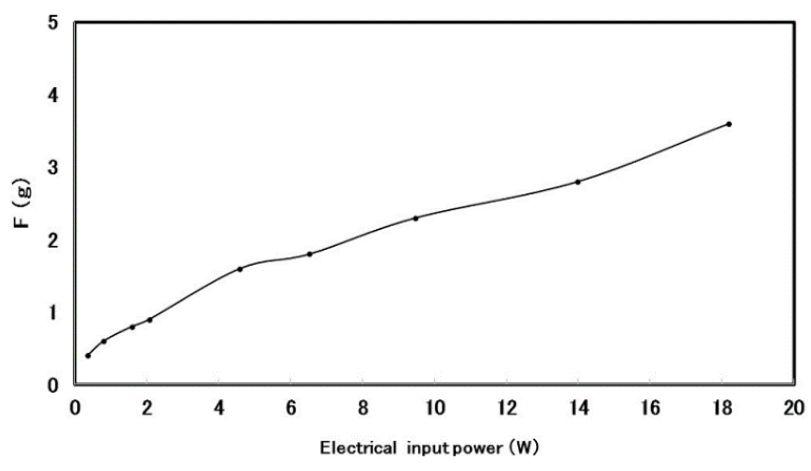

(b)

Fig. 12. Measured propulsion force results for model B2. (a) Voltage-force property, (b) Power-force property.

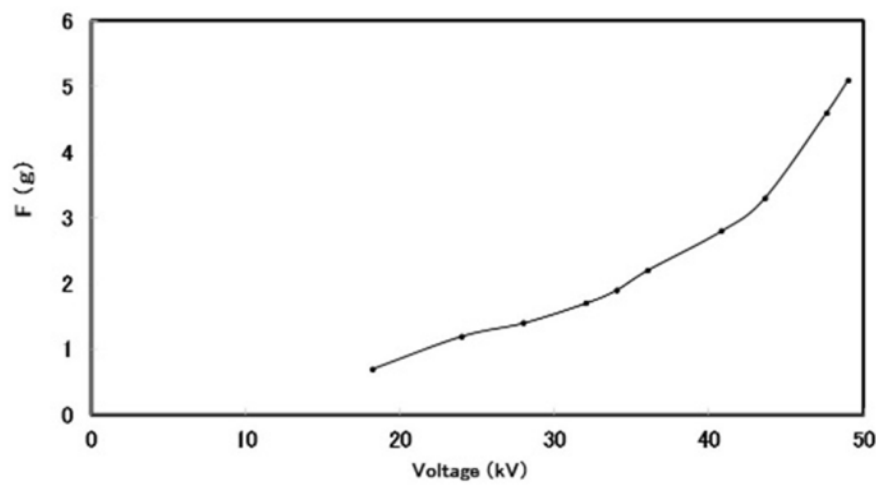

(a)

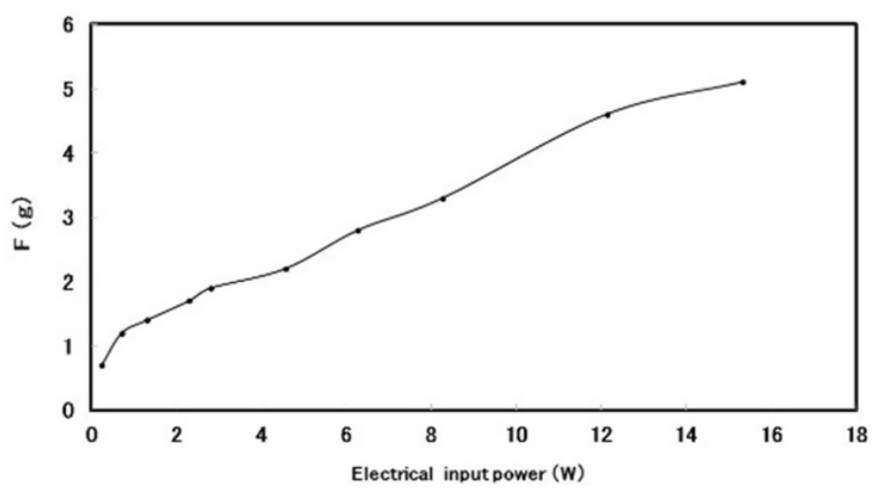

(b)

Fig. 13. Measured propulsion force results for model B3. (a) Voltage-force property, (b) Power-force property.

\section{Discussion}

I proposed modified EHD and electrostatic devices to improve the ratio of the propulsion force to electrical input power. In the numerical analysis, the results of the calculated total charge on the surface of the large electrode were enlarged due to the polarization effect. From the propulsion force results obtained for the devices governed by eq. (1) and eqs. (13) (14), because the forces are proportional to the charges on the surface of the large flat electrodes, it can be concluded that the polarization effect improves the forces.

The calculated results lead one to expect that the force that model B3 generates will be 7 times higher than that of model Aa1 owing to eq. (1) and eq. (13). If the devices are operated at a high voltage, the gap used will be longer, the stored charges on the large and flat electrodes will be larger, and the quantity of the charges will be enlarged proportional to the gap length approximately.

Table 3. Comparison of measured and calculated propulsion force.

\begin{tabular}{|c|c|c|c|c|c|c|c|c|}
\hline Model & Voltage $(\mathrm{kV})$ & Current (mA) & Power $(W)$ & $F \exp (g)$ & d (m) & Fcal(g) Eq.(3) & $\alpha$ Fexp/ Fcal & $\theta(\mathrm{N} / \mathrm{kW})$ \\
\hline $\mathrm{Aa} 2$ & 42 & 0.15 & 9.9 & 1.8 & 0.09 & 1.1 & 1.6 & 1.8 \\
\hline $\mathrm{Ab}$ & 45 & 0.39 & 19 & 2.8 & 0.09 & 1.7 & 1.6 & 1.4 \\
\hline B1 & 46 & 0.31 & 14 & 2.2 & 0.066 & 0.94 & 2.4 & 1.6 \\
\hline B3 & 48 & 0.26 & 12 & 4.6 & 0.066 & 0.81 & 5.7 & 3.8 \\
\hline
\end{tabular}

In the experiment on the forces that model $\mathrm{A}$ and $\mathrm{B}$ generated, the models showed high performance with respect to the ratio of force to electrical input power.

Especially for model B3, A large propulsion force are generated whenever ion flows in the horizontal direction. The ion wind cannot be contribute to the enhanced propulsion force. This should prove that the lifter work by the electrostatic propulsion as mentioned in the reference[6,7].

The electrical input power, measured generated power, calculated generated force, and ratio are shown in Table 3.
The gap length $\mathrm{d}$ is the value for which $1 \mathrm{~cm}$ was added to the gap length from the edge of the radial electrode to the negative needle electrode in Table 3. I used eq. (3) to calculate the theoretical values of the propulsion force the devices generated. The ion mobility was set to be $2.15 \times 10^{-4}\left(\mathrm{~m}^{2} / \mathrm{Vs}\right)$.

The ratio of generated force to electrical input power of a basic lifter with a single narrow electrode and a single flat aluminum electrode, as shown in reference [1,2], was estimated to be $1.3 \mathrm{~N} / \mathrm{kW}$.

It has been clarified from this experiment that the total 
amount of ions generated from the small electrode in the conventional lifter model is large. For a large, flat electrode, however, the ion density is low.

The $0.4 \mathrm{~mA}$ current for model Aa2 with $16-\mathrm{cm}$ outer diameter is the same as that of the basic lifter at the input voltage of $46 \mathrm{kV}$. However, the generated force for model Aa2 is 1.6 times higher than that of the basic lifter as shown in Table 3. The model A structure with downstream ion flows was able to confine ions generated from the negative electrode and prevent the ion density from being low close to the large radial electrode.

The forces that the models B1, B2, and B3 generated are rigidly proportional to the square of the input voltage because the electric field intensity increases when the number of ions generated increases at the same time.

The forces that model Aa generated were rigidly generated in proportion to the input voltage because the electric field intensity is constant when the number of generated ions increases.

The forces that models Aa1 and Aa2 generated saturated when the input voltage was $43 \mathrm{kV}$. This is because the generated ions were saturated in the air, the ion density consequently reached the space charge limit, and the ions were repelled near the large electrode.

For model $\mathrm{Ab}$, the inner ring electrode was set in the center of the large ring electrode and the measured generated force was more than twice that measured for model Aa2. The reason is that the surface area of the positive large electrode increased. However, a model having too many ring electrodes will not result in an increase in the generated force.

For model B1 using the polarization effect, the ring electrodes were cascaded and the force measured for it was 3 times as large as that measured for model Aa1.

For model B2 using the polarization effect, there was a pole to generate polarization. The force measured for model B2 was close to 2 times larger than that measured for model Ac.

The force measured for model B3 was $4.6 \mathrm{~g}$ at an input voltage of $46 \mathrm{kV}$ and electrical input energy of $12 \mathrm{~W}$. The ratio of the generated force to electrical power was $4 \mathrm{~N} / \mathrm{kW}$. The value was maximum in all the proposed models. The current of model B3 was $50 \%$ lower than that of model Aa1 for the same input voltage of $24 \mathrm{kV}$. The ratio of the generated force to electrical input power was $15 \mathrm{~N} / \mathrm{kW}$ at $23 \mathrm{kV}$ input voltage. This is one order higher than that of the basic lifter, for which the ratio was $8.3 \mathrm{~N} / \mathrm{kW}$ at $28 \mathrm{kV}$ input voltage. The generated force for input voltage was not saturated. The property is ideal and well consistent with the theory. The polarization plate electrodes should be set to be tilted for the large rounded electrode. The maximum angle for generating force should exist. In these experiments, I was able to modify the ratio of the generated force to the electrical input power.

However, the ratio of the generated force to unit weight has not been modified yet. Using a high voltage generator with higher output voltage and longer gap length will enable the ratio of generated force to unit weight to be improved. Specially, the ratio of the models B1, B2, and B3 should be substantially modified. The aluminum foil thickness used in the experiment was $10 \mu \mathrm{m}$; this thickness should have an optimized value.

If the aluminum foil is very thin, the potential that confines the charge will degrade and thus charges on the metal plate cannot be confined efficiently. Basically, the structure of the large electrode must be maintained to store a lot of charges. In the future, the optimized thickness should be studied.

Modifications should also be made to obtain a higher ratio of the generated force to electrical input power. Using multiple large, flat electrodes as positive electrodes should make it possible to improve the ratio, since it should enhance the electrical field intensity. The experimental data in reference [12] shows the effect to improve the ratio of generated force to electrical input power clearly. However, the effects of enhancing the electric field intensity due to the edge and flat surfaces of electrodes are mixed. Thus, we cannot divide them. In reference [12], a $6.5 \mathrm{~N} / \mathrm{kW}$ ratio was obtained when the small electrode was negative $s$ in a high voltage and high power regime. The models I showed may be optimized, in which case it should be able to obtain a higher ratio than this. It is hard to set B2 modules in cascade but it is possible to set them in parallel. Models B1 and B3 can be set in series. Setting devices in series, as shown at right in Fig. 3, will result in an increased ratio of generated force to electrical input power. Using a multi-electrode structure comprising a large flat electrode, multi-stage polarization electrodes, and using long gap between electrodes and more high input voltage [13] should make it possible to raise the ratio to over $100 \mathrm{~N} / \mathrm{kW}$ at a low electrical input power and less $100 \mathrm{~N} / \mathrm{kW}$ at a high electrical input power. Using long gap between electrodes results in improving the ratio of generated force to electrical input power as shown in eq. (4) because the electric field is weak for the same input voltage [13]. The ratio at a high electrical input power would be higher than that of a conventional helicopter using a motor, for which the ratio is around $70 \mathrm{~N} / \mathrm{kW}$.

Combining model B3 with model B1 will result in generating a force of over $10 \mathrm{~g}$. Moreover, using weakly ionized plasma to produce ultra-high voltage, as in EHD generators [15], we should use the optimized system to reduce the weight of the high voltage power supply and improve the ratio of generated force to electrical input power.

\section{Conclusion}

This paper discussed various kinds of proposed EHD and electrostatic propulsion devices using asymmetrical polarization electrodes that I propose as means to improve the ratio of generated propulsion force to electrical input power. The levels of force that the devices generated were measured in experiments. Electrical charges on the surfaces of electrodes were estimated numerically with an aim to improving the generated force. The models substantially improved the generated force; for the same electric energy, the force they generated was 5.7 times higher than that of a basic type lifter. This was due to additional propulsion force being generated by the polarization effect. 
Future subjects in this research will include using 1) a high voltage supply with reduced weight achieved by using a flame-jet or low-density-plasma EHD generator and 2) multiple polarization electrodes as means to improve the generated propulsion force.

\section{References}

[1] T. T. Brown, "Electrokinetic Apparatus," U.S. Patent N²949550, 1960.

[2] http://www.jlnlab.com/

[3] http://www.blazelabs.com/

[4] M. Tajmar, "Biefeld-Brown Effect: Misinterpretation of Corona Wind Phenomena", AIAA Journal 42(2) (2004).

[5] L. Zhao and K. Adamiak, "Numerical analysis of forces in an electrostatic levitation unit," J. of Electrostatics, 63, pp. 729-734 (2005).

[6] L. Zhao, K. Adamiak, "EHD gas flow in electrostatic levitation unit”, J. of Electrostatics, 64, pp. 639-645 (2006).

[7] A. A. Martins, M. J. Pinheiro, "Modeling of an EHD corona flow in nitrogen gas using an asymmetric capacitor for propulsion”, J. of Electrostatics, 69 (2), pp. 133-138 (2011).
[8] R. Ianconescu, D. Sohar, and M. Mudrik, "An analysis of the Brown- Biefeld effect," J. of Electrostatics, 69, pp. 512-521 (2011).

[9] M. Chen, L. Rong-de, Y. Bang-jiao, "Surface aerodynamic model of the lifter," J. of Electrostatics, 71(2), pp.134-139 (2013).

[10] F. X. Canning, C. Melcher, and E. Winet, "Asymmetrical Capacitors for Propulsion," NASA, NASA/CR - 2004-213312 (2004).

[11] J. Wilson, H. D. Perkins, and W. K. Thompson, "An Investigation of Ionic Wind Propulsion," NASA, NASA/TM-2009-215822 (2009).

[12] M. Eiant and R. Kalderon, AIP advances, 4, 077120-1-20 (2014).

[13] K. Masuyama and S. R. H. Barret, "On the performance of electro hydrodynamic propulsion", Proc. of Royal Soc. A, 469 (2154), 20120623-1-16 (2013).

[14] T. Kouno, "High Voltage Engineering", Asakura Publishing, Tokyo (1995) (Chapter 2, in Japanese).

[15] T. Saiki, "Study on High Voltage Generation Using Flame Column and DC Power Supply", J. of Electrostatics, 70, pp.400-406 (2012). 